\title{
Sleep Quality in Rheumatoid Arthritis: Relationship Between the Disease Severity, Depression, Functional Status and the Quality of Life
}

\author{
Mustafa Akif Sariyildiz ${ }^{\mathrm{a}, \mathrm{e}}$, Ibrahim Batmaz ${ }^{\mathrm{a}}$, Mehtap Bozkurta, Yasin Bez ${ }^{\mathrm{b}}$, \\ Mehmet Guli Cetincakmak ${ }^{\mathrm{c}}$, Levent Yazmalara, \\ Demet Ucar ${ }^{\mathrm{a}}$, Tahsin Celepkolu ${ }^{\mathrm{d}}$
}

\begin{abstract}
Background: The aim of this study was to evaluate sleep quality and the related variables in patients with rheumatoid arthritis (RA).

Methods: Ninety-four patients diagnosed with RA and fifty two healthy controls were enrolled in the study. Disease activity was assessed through the Disease Activity Score (DAS) 28 scale. All patients were assessed using the Rheumatoid Arthritis Quality of Life and Health Assessment Questionnaire scales, together with the Beck Depression Inventory. Radiological damage was calculated with the modified Larsen method. The Pittsburgh Sleep Quality Index (PSQI) was used for the evaluation of the sleep disturbance.
\end{abstract}

Results: The patients with RA had significantly higher scores in the subjective sleep quality, sleep latency, habitual sleep efficiency, sleep disturbance domains and the total PSQI score compared to the healthy control group. According to the results of Spearman's analysis, there was a significantly correlation between the age, disease activity, CRP, pain, fatigue, depression, functional disability, quality of life, radiological damage, menopause status, duration of morning stiffness, ESR levels and the sleep disturbance. The logistic regression analysis indicated that depression and DAS 28 scores were predictors for poor sleep quality.

Conclusion: The sleep quality is disturbed in patients with RA. The poor sleep quality is especially associated with the disease activity

\footnotetext{
Manuscript accepted for publication October 24, 2013

${ }^{a}$ Department of Physical Medicine and Rehabilitation, Dicle University Faculty of Medicine, Diyarbakir, Turkey

${ }^{\mathrm{b}}$ Department of Psychiatry, Dicle University Faculty of Medicine, Diyarbakir, Turkey

${ }^{\mathrm{c}}$ Department of Radiology, Dicle University Faculty of Medicine, Diyarbakir, Turkey

${ }^{\mathrm{d}}$ Department of Family Physician, Dicle University Faculty of Medicine, Diyarbakir, Turkey

${ }^{\mathrm{e}}$ Corresponding author: Mustafa Akif Sariyildiz, Department of Physical Medicine and Rehabilitation, Dicle University Faculty of Medicine, Diyarbakir, Turkey. Email: makifsariyildiz@hotmail.com
}

doi: http://dx.doi.org/10.4021/jocmr1648w and depression.

Keywords: Rheumatoid arthritis; Sleep quality; Disease activity; Depression

\section{Introduction}

Rheumatoid arthritis (RA) is a chronic autoimmune disorder characterized by joint pain, joint swelling, morning stiffness, fatigue, depression, sleep disturbances and disability [1-4]. Several studies that have pointed out sleep disturbances in RA patients have revealed that $54 \%$ to $70 \%$ of RA patients report problems related to their sleep, including difficulty falling asleep, poor sleep quality, non restorative sleep, wakefulness, awakenings during the night and excessive daytime sleepiness [5-8]. Conversely, sleep disturbance may contribute to the pain, disease activity, stiffness, fatigue and mood disturbances in affected patients [7, 9-11].

Possible causes of sleep disturbance in RA include inflammatory disease activity, joint pain, sleep apnea, restless leg syndrome and psychological disorders like depression $[2$, 12]. Wolfe et al [12] have revealed that pain and depression are independently associated with sleep disturbances in RA. Nicassio and Wallston [13] have shown that sleep disturbance is associated with depression independently of pain, and that long-term pain is a predictor of deteriorating sleep disorders.

Sleep quality is an important component of the quality of life, and it is a known fact that sleep disturbances affect the quality of life in patients with RA [14]. It has also been demonstrated that the higher depression, fatigue and pain scores in patients with RA are related to functional disability $[15,16]$ Wolfe et al $[16]$ have revealed that pain and depression are important determinants of the functional disability in patients with RA. Poor sleep quality may contribute to the feelings of pain, fatigue and mood disturbances and may further deteriorate the functional ability and the quality of life.

The aim of this study is to determine the influence of RA on sleep quality and to reveal the relationship of the laboratory parameters, radiological damage, disease activity, func- 
tional status, quality of life and the psychological state with the sleep disturbance.

\section{Material and Method}

\section{Participants}

Ninety-four patients who applied to the Physical Medicine and Rehabilitation outpatient clinic in university hospital in Turkey between October 2011 and May 2012 and diagnosed with RA according to the 1987 American College of Rheumatology [17] criteria were included in this study. The patients were between the ages of 18 and 65. All the patients were married and have regularly been using DMARD and/ or anti-TNF for the last three months. Fifty two age- and sex-matched healthy subjects were also enrolled as the control group. The present study had a cross-sectional design approved by the local ethics committee. All the recruited subjects signed an informed consent form before participating in the study.

Patients with thyroid disorders, infections, those who had undergone a hysterectomy or who had any chronic diseases such as diabetes mellitus, heart or renal failure, as well as those with a history of serious psychiatric disorders were excluded from study.

\section{Measurement of the clinical variables}

The demographic and clinical characteristics of the patients such as age, sex, height, weight, educational level, the disease duration (which is defined as the duration since the onset of the first symptoms of RA), degree of morning stiffness, tender-swollen joint count and the physician's and patient's global assessments were determined. Drug use (Non steroidal anti-inflammatory drugs (NSAIDs), diseasemodifying anti-rheumatic drugs (DMARDs), anti-tumour necrosis factor (TNF), or corticosteroids) and the smoking status were recorded. The duration of the morning stiffness (minutes), mean pain and mean daytime fatigue $(100 \mathrm{~mm}$ visual analogue scale (VAS)) were also noted. The disease activity (DAS28) scores were evaluated using the disease activity score calculator [18]. The erythrocyte sedimentation rate (ESR) was measured through the Westergren method $(\mathrm{mm} / \mathrm{h})$ and the serum C-reactive protein (CRP) level was determined with the help of nephelometry $(\mathrm{mg} / \mathrm{dL})$. The RF titres were also measured through the nephelometric immunoassay method (IU/mL).

Functional disability, radiological score and the quality of life

The quality of life was evaluated using the disease-specific rheumatoid arthritis quality of life (RAQoL) scale [19]. The health assessment questionnaire (HAQ) was used for the evaluation of the functional status [20]. Standardized radiographs of both the hands and feet were performed for all the RA patients and the degree of joint damage were assessed by a radiologist according to Larsen's method [21].

\section{Measurements of the psychological variables}

The Beck depression inventory (BDI) was used for evaluation of the depression in patients with RA. BDI is a likerttype, self-reported questionnaire which evaluates the level of depression according to the replies to 21 questions [22]. In our study, a BDI score of 13 was chosen as the cut-off point for depression as recommended [23]. The validity and reliability of the scale's Turkish version have already been shown as satisfactory [24]. The results were evaluated by a psychiatrist.

\section{Measurement of sleep quality}

The Pittsburgh Sleep Quality Index (PSQI) measures the patient's self-reported sleep quality over the last month [25]. The scale has 19 items and measures 7 components of sleep quality: subjective sleep quality, sleep latency, sleep duration, habitual sleep efficiency, sleep disturbances, use of sleeping medication, and daytime dysfunction. A global PSQI score corresponding to the total of the individual scores from the 7 components is calculated (range $=0-21$ ). A result equal to 6 or above is considered indicative of poor sleep quality. The test has high diagnostic specificity for detecting clinical sleep impairment. The Turkish version of this scale has been verified in terms of its validity and reliability by Agargun et al [26].

\section{Statistical analysis}

The calculations were performed using the Statistical Package for Social Sciences for Windows software version 16.0. The Kolmogorov-Smirnov test was used to confirm that data within the ranges of normal distribution in both groups. A non-parametric test was employed for the variables outside the normal distribution. The comparison of the data between reciprocal groups was carried out through the Mann Whitney$\mathrm{U}$ test. The independent-sample t-test was used to examine the differences in sleep quality, pain, depression, fatigue, and functional disability between those taking certain RA medications and those who do not. Correlations between sleep disturbance, depression status and the RA-related variables were investigated with the help of Spearman's correlation. By using a binary logistic regression analysis, a multivariate analysis was performed to detect independent predictors for the occurrence of sleep disturbances and to find out confounding effects between potentially independent predictors. A forward stepwise method was used to construct multivari- 
Table 1. Clinical and Demographic Characteristics of the Patients With RA and Healthy Controls (Mean \pm SD)

\begin{tabular}{llll}
\hline Disease characteristics & $\begin{array}{l}\text { RA patients } \\
(\mathbf{n : 9 4 )}\end{array}$ & $\begin{array}{l}\text { Controls } \\
(\mathbf{n}: 52)\end{array}$ & P \\
\hline Age (years) & $46.20 \pm 12.32$ & $44.07 \pm 9.75$ & NS \\
Female & $81.5 \%$ & $79.2 \%$ & NS \\
Postmenopausal patient & $48 \%$ & $45 \%$ & NS \\
Disease duration (years) & $4.66 \pm 5.14$ & \\
Duration of morning stiffness (minute) & $62.99 \pm 57.78$ & \\
Pain (VAS, mm) & $5.14 \pm 2.65$ & \\
Fatigue (VAS, mm) & $4.68 \pm 2.56$ & \\
ESR (mm/h) & $29.18 \pm 21.71$ & \\
CRP (mg/dL) & $3.28 \pm 6.26$ & \\
RF (IU/mL) & $120.57 \pm 176.25$ & \\
HAQ & $1.08 \pm 0.87$ & \\
DAS28 & $4.17 \pm 1.59$ & \\
Depression (BDI score) & $15.56 \pm 8.75(53.3 \%)$ & \\
RAQoL & $14.05 \pm 10.19$ & \\
Larsen score & $30.47 \pm 19.70$ & \\
NSAID & $62 \%$ & \\
Anti-TNF & $48 \%$ & \\
DMARD & $72 \%$ & \\
Corticosteroid & $42 \%$ & \\
\hline
\end{tabular}

VAS: visual analog scale; ESR: Erythrocyte sedimentation rate; CRP: C-reactive protein; RF: rheumatoid factor; HAQ: health assesment questionnaire; DAS: disease activity score; BDI: Beck depression inventory; RAQoL: rheumatoid arthritis quality of life; RA: rheumatoid arthritis; n: number of cases; SD: standard deviation.

ate logistic regression models in relation to various dependent variables. Statistical significance was based on a value of $\mathrm{P}<0.05$ with a $95 \%$ confidence interval.

\section{Results}

The clinical and demographic characteristics of the patients and the healthy controls are listed in Table 1 . The majority of the RA patients were female (81.5\%). The mean age was $46.20 \pm 12.32$ years. According to the suggested cut off point for the PSQI global score (scores > 5), sleep disturbances were observed in $64.1 \%$ of the patients with RA. Among the patients, $43.3 \%$ had depression symptoms (BDI > 13) and $48 \%$ were postmenopausal. The patients with RA had significantly higher scores in the subjective sleep quality, sleep latency, habitual sleep efficiency, sleep disturbance domains and in terms of the total PSQI score compared to the healthy control group $(\mathrm{P}<0.05)$ (Table 2).

According to Spearman's analysis, there was a significantly higher correlation between the age, pain, fatigue, DAS28, CRP, BDI, HAQ, RAQoL, the Larsen scores and the sleep disturbance $(\mathrm{P}<0.01)$. There was also a significantly lower correlation between the menopause status, duration of morning stiffness, ESR levels and the sleep disturbance (P $<0.05)$. Correlations between the disease-related variables, 
Table 2. Mean Scores of the RA Patients and Controls for the PSQI Domains (Mean \pm SD)

\begin{tabular}{llll}
\hline & $\begin{array}{l}\text { RA Patients } \\
(\mathbf{n : 9 4 )}\end{array}$ & $\begin{array}{l}\text { Controls } \\
(\mathbf{n : 5 2})\end{array}$ & $\mathbf{P}$ \\
\hline Subjective sleep quality & $1.61 \pm 0.86$ & $0.81 \pm 0.56$ & $<0.001$ \\
Sleep latency & $1.36 \pm 0.96$ & $0.77 \pm 0.73$ & $<0.001$ \\
Sleep duration & $1.14 \pm 0.87$ & $1.00 \pm 0.65$ & 0.274 \\
Habitual sleep efficiency & $0.73 \pm 0.99$ & $0.21 \pm 0.63$ & $<0.001$ \\
Sleep disturbances & $1.24 \pm 0.81$ & $1.00 \pm 0.56$ & 0.040 \\
Use of sleeping medication & $0.10 \pm 0.38$ & $0.11 \pm 0.42$ & 0.925 \\
Daytime dysfunction & $0.80 \pm 0.78$ & $0.73 \pm 0.69$ & 0.560 \\
PSQI total & $7.03 \pm 3.97$ & $4.60 \pm 2.64$ & $<0.001$ \\
\hline
\end{tabular}

SD: standard deviation; PSQI: Pittsburgh sleep quality index; RA: rheumatoid arthritis; n: number of cases.

individual domains and the total PSQI scores are listed in Table 3.

When the patients were compared in terms of the medication status (anti-TNF, DMARD, corticosteroids, or none), no significant difference has been observed between the treatment modality and the PSQI scores $(\mathrm{P}>0.05)$. The logistic regression analyses indicated that BDI and DAS 28 scores were predictors for poor sleep quality $(\mathrm{P}<0.05)$ (Table 4).

\section{Discussion}

In this study, the mean PSQI scores indicate poorer sleep quality in the patients with RA compared to the controls. The logistic regression analyses have pointed out the disease activity and depression as the predictors of poor sleep quality. In various studies conducted in patients with RA, depression has been reported to have a negative influence on the quality of sleep and it has also been described as a risk factor for sleep quality $[9,12,13,27]$. The pain in the patients with RA leads to sleep disturbances, while the constant pain and sleep disturbances increase the patient's propensity to depression $[13,28]$. The multivariate regression analyses performed in a longitudinal study by Nicasso et al [13] have demonstrated that severe pain and severe sleep disorders are independently associated with depression in the patients with RA. These results are also in line with our study and point out that the depression contributes to sleep disorders in patients with RA.

In previous studies, pain at rest and joint stiffness were determined to be factors associated with sleep disturbances $[5,13,15,29]$. It has also been reported that the duration of morning stiffness affects the quality of sleep [7, 15, 30, 31]. In a recent study, Luyster et al [3] found that poorer sleep quality is associated with greater pain severity. Aside from these findings, it has also been shown that the sleep quality is also positively influenced when the active inflammatory disease and the resulting pain are treated and the arthritic process is stopped $[32,33]$. Many investigators have observed that any increase in the disease activity is related with sleep disorders in patients with RA [7, 12, 34, 35]. There is also a study where the disease activity was found to be unrelated with sleep disorders, but this conflicting result may be due to the small number of patients that participated in the study $(\mathrm{n}=19)$ [8]. The DAS 28 score used to measure the disease activity in the patients with RA is related to the number of the tender and swollen joints. A greater number of tender and swollen joints may cause frequent interruptions during the sleep throughout the night and lead to longer durations of morning stiffness (in our study, the mean duration of morning stiffness was 62 minutes). Consequently, since getting up and moving around may get problematic, the sleep quality is negatively affected. Also in our study, the disease activity, greater pain, and morning stiffness were found to be associated with sleep disorders.

Mahowlad et al [36] explored sleep in RA patients using polysomnography and suggested that fatigue in RA may be a manifestation of sleep fragmentation. Similarly, sleep quality has also been recognized as a predictor of fatigue in older adults with RA [15]. In a recent study, Fuyster et al [3] revealed that poor sleep quality is associated with higher levels of fatigue. In patients with RA, while the fatigue may disturb sleep quality, the disturbance in the sleep quality may also lead to greater fatigue [37]. In accordance with these 


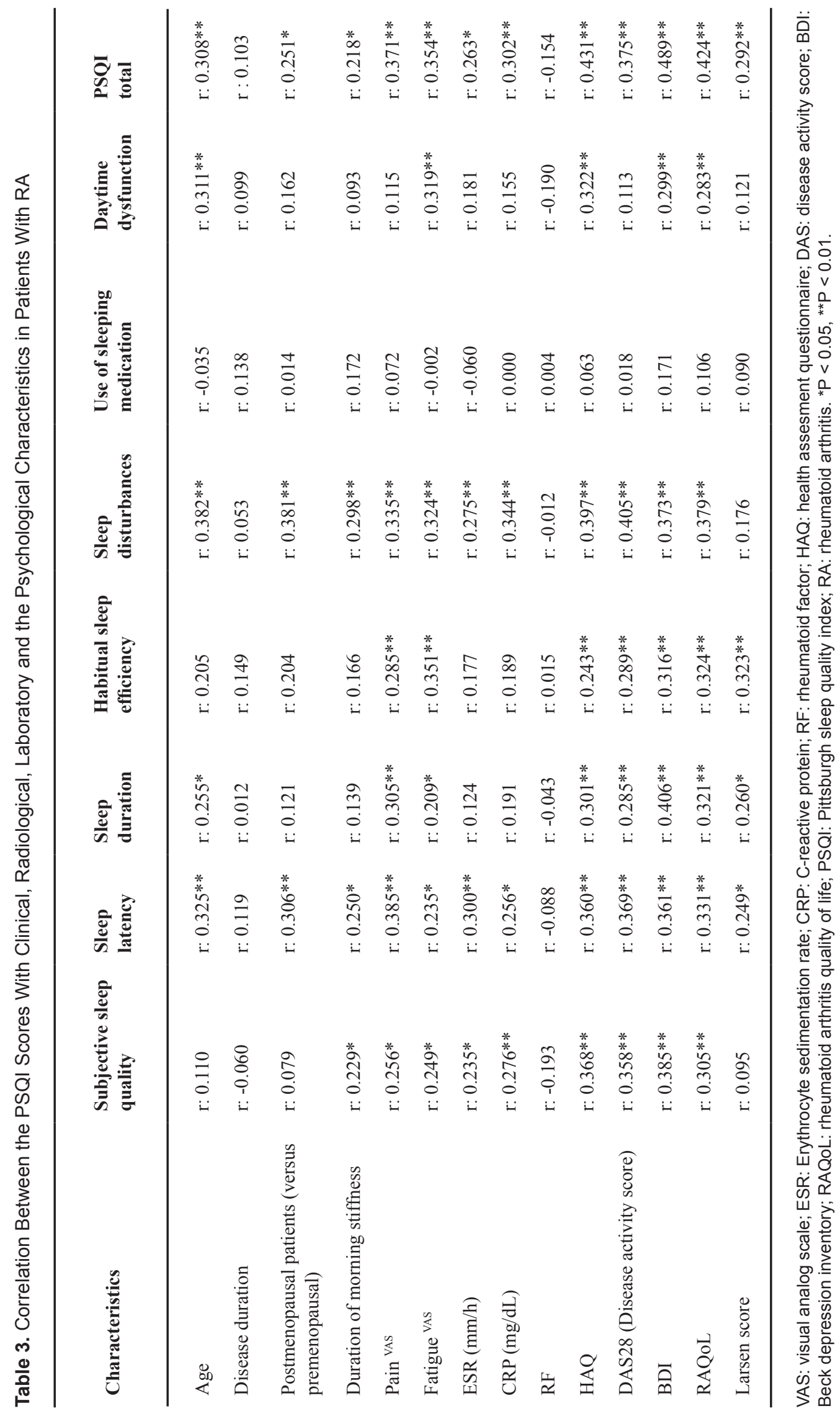


Table 4. Final Logistic Regression Model; PSQI Total Score as Dependent Variable (PSQI $\geq 6$ Versus PSQI $<6)$

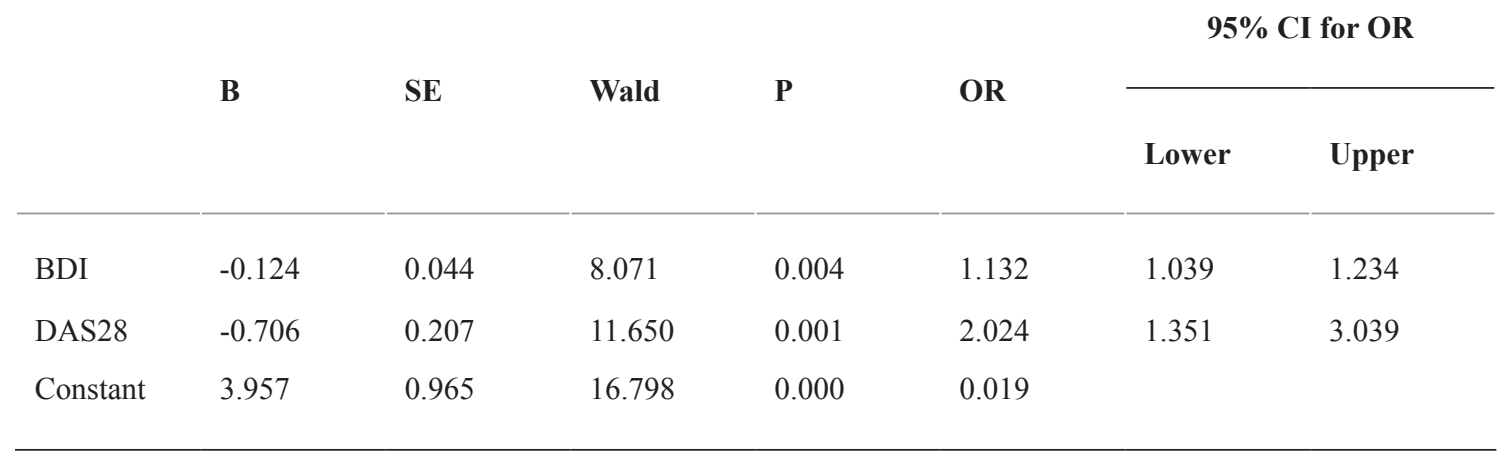

DAS: disease activity score; BDI: Beck depression inventory; B: coefficients; SE: standard error; OR: odds ratio; CI: confidence interval.

studies, also in the present study, higher fatigue levels were observed to be associated with sleep disturbance according to the PSQI index.

In this study, a higher Larsen score was also correlated with poor sleep quality. The association of the radiological damage with the sleep quality were not investigated in the previous studies. On the other hand, there are various studies focussing on the relationship between the radiological damage, and the disability and quality of life in the patients with RA. Some studies have pointed out that the influence of the radiological damage on the quality of life was less severe compared to pain, depression, disease activity and the functional status $[38,39]$. At the same time, Prajs et al [40, 41] have suggested that radiological damage and functional status affect the quality of life in RA more than the disease duration and disease activity. In accordance with these results, our study revealed the influence of the radiological damage on poor sleep quality in RA patients. Since radiological damage is associated with the disease activity and the low quality of life, it may contribute to sleep disturbances.

Higher RAQoL and higher HAQ scores were also correlated with sleep disturbances in the present study. Many patients with RA experience difficulties in the activities related to daily life. This causes greater fatigue during the day, which leads to interrupted sleep at night, thus to sleep disturbances. Also, the inability to perform the daily activities may cause depression and lead to sleep disturbance in patients with RA [42]. In RA populations, fatigue, pain and depression have been found to be associated with the physical disability $[15$, 42]. Wolfe et al [16] have assessed functional disability using the Health Assessment Questionnaire (HAQ) and found pain and depression to be important determinants of functional disability in 1843 patients with RA. Based on the links between sleep, pain, depression and disability; an indirect pathway may exist between the sleep quality and functional disability in RA [3]. The causal relationships between pain, fatigue, depression and disability may be reciprocal. While the pain, fatigue and depression may interfere with the activities related to daily life and thus cause sleep disorders and disability, sleep disorders may also contribute to the pain, fatigue and depression and lead to disability [3].

In our study, Spearman's analyses revealed that the postmenopausal status of the patients was correlated with the sleep disturbances. Also, 78\% of the postmenopausal RA patients described hot flashes. Sleep quality may also be disturbed in postmenopausal women. Approximately $70 \%$ of healthy women experience hot flashes. Hot flashes occur as a result of the increase in the plasma epinephrine and brain norepinephrine levels besides other autonomic changes such as increased heart rate, palpitations, and feelings of anxiety or panic $[43,44]$. Healthy women who experience hot flashes have more frequent sleep disturbances [45].

The relationship between the medication and sleep disorders in RA is unclear. Placebo controlled studies have demonstrated that NSAIDs increase sleep quality [46, 47]. However, polysomnographic results have indicated that the administration of NSAIDs does not change the sleep architecture, sleep latency or sleep efficiency in patients with RA [48]. However, there are also studies showing that anti-TNF agents increase the sleep quality in patients with RA [49, 50]. In their prospective and controlled study, Wells et al [49] have reported greater improvement in patients using abatecept in comparison to the patients using methotrexate. In the present study, no relationship was detected between the RA medication and sleep quality.

We have observed the sleep disorder to be positively correlated with the patients' age in our study. The relationship of the age with the sleep quality in the patients with RA is yet to be cleared. Bixler et al [51] have reported that the age is correlated with shorter sleep durations. In another study, the sleep quality in patients with OA and RA was compared and the sleep disorders in patients with OA were attributed 
to the age [27]. Still, some studies have failed to reveal any relationship between the age and the sleep disorder [3, 9].

The limitation of this study was its cross-sectional design. Prospective studies are needed in order to reveal the relationship between disease activity, depression, pain, functional disability and sleep quality. Besides, we have evaluated the sleep quality through a questionnaire, while a polysomnographic evaluation could have supplied more accurate results.

\section{Conclusion}

As far as we know, this is the first study evaluating correlation of the radiological scores and the sleep quality in patients with RA. Also, the effect of the disease activity and depression on the sleep quality has been demonstrated by means of the logistic regression model in this study. Poor sleep quality was observed in RA patients. Sleep disturbances in patients with RA occur due to multifactorial origins and multidirectional disease-related variables like the disease activity, depression, pain, fatigue, functional disability, radiographic score, quality of life and the CRP levels.

\section{Conflicts of Interest}

The authors report no conflicts of interest.

\section{References}

1. Escalante A, Del Rincon I. The disablement process in rheumatoid arthritis. Arthritis Rheum. 2002;47(3):333342 .

2. Abad VC, Sarinas PS, Guilleminault C. Sleep and rheumatologic disorders. Sleep Med Rev. 2008;12(3):211228.

3. Luyster FS, Chasens ER, Wasko MC, Dunbar-Jacob J. Sleep quality and functional disability in patients with rheumatoid arthritis. J Clin Sleep Med. 2011;7(1):49-55.

4. Kojima M, Kojima T, Ishiguro N, Oguchi T, Oba M, Tsuchiya H, Sugiura F, et al. Psychosocial factors, disease status, and quality of life in patients with rheumatoid arthritis. J Psychosom Res. 2009;67(5):425-431.

5. Drewes AM, Nielsen KD, Hansen B, Taagholt SJ, Bjerregard K, Svendsen L. A longitudinal study of clinical symptoms and sleep parameters in rheumatoid arthritis. Rheumatology (Oxford). 2000;39(11):1287-1289.

6. Moldofsky H, Lue FA, Smythe HA. Alpha EEG sleep and morning symptoms in rheumatoid arthritis. J Rheumatol. 1983;10(3):373-379.

7. Drewes AM, Svendsen L, Taagholt SJ, Bjerregard K, Nielsen KD, Hansen B. Sleep in rheumatoid arthritis: a comparison with healthy subjects and studies of sleep/ wake interactions. Br J Rheumatol. 1998;37(1):71-81.

8. Hirsch M, Carlander B, Verge M, Tafti M, Anaya JM, Billiard M, Sany J. Objective and subjective sleep disturbances in patients with rheumatoid arthritis. A reappraisal. Arthritis Rheum. 1994;37(1):41-49.

9. Nicassio PM, Ormseth SR, Kay M, Custodio M, Irwin MR, Olmstead R, Weisman MH. The contribution of pain and depression to self-reported sleep disturbance in patients with rheumatoid arthritis. Pain. 2012;153(1):107112.

10. Moldofsky H. Sleep and pain. Sleep Med Rev. 2001;5(5):385-396.

11. Hamilton NA, Catley D, Karlson C. Sleep and the affective response to stress and pain. Health Psychol. 2007;26(3):288-295.

12. Wolfe F, Michaud K, Li T. Sleep disturbance in patients with rheumatoid arthritis: evaluation by medical outcomes study and visual analog sleep scales. J Rheumatol. 2006;33(10):1942-1951.

13. Nicassio PM, Wallston KA. Longitudinal relationships among pain, sleep problems, and depression in rheumatoid arthritis. J Abnorm Psychol. 1992;101(3):514-520.

14. Cappuccio FP, D'Elia L, Strazzullo P, Miller MA. Sleep duration and all-cause mortality: a systematic review and meta-analysis of prospective studies. Sleep. 2010;33(5):585-592.

15. Belza BL, Henke CJ, Yelin EH, Epstein WV, Gilliss CL. Correlates of fatigue in older adults with rheumatoid arthritis. Nurs Res. 1993;42(2):93-99.

16. Wolfe F. A reappraisal of HAQ disability in rheumatoid arthritis. Arthritis Rheum. 2000;43(12):2751-2761.

17. Arnett FC, Edworthy SM, Bloch DA, McShane DJ, Fries JF, Cooper NS, Healey LA, et al. The American Rheumatism Association 1987 revised criteria for the classification of rheumatoid arthritis. Arthritis Rheum. 1988;31(3):315-324.

18. Prevoo ML, van't Hof MA, Kuper HH, van Leeuwen MA, van de Putte LB, van Riel PL. Modified disease activity scores that include twenty-eight-joint counts. Development and validation in a prospective longitudinal study of patients with rheumatoid arthritis. Arthritis Rheum. 1995;38(1):44-48.

19. Kutlay S, Kucukdeveci AA, Gonul D, Tennant A. Adaptation and validation of the Turkish version of the Rheumatoid Arthritis Quality of Life Scale. Rheumatol Int. 2003;23(1):21-26.

20. Kucukdeveci AA, Sahin H, Ataman S, Griffiths B, Tennant A. Issues in cross-cultural validity: example from the adaptation, reliability, and validity testing of a Turkish version of the Stanford Health Assessment Questionnaire. Arthritis Rheum. 2004;51(1):14-19.

21. Larsen A. How to apply Larsen score in evaluating radiographs of rheumatoid arthritis in long-term studies. J Rheumatol. 1995;22(10):1974-1975. 
22. Beck AT, Ward CH, Mendelson M, Mock J, Erbaugh J. An inventory for measuring depression. Arch Gen Psychiatry. 1961;4:561-571.

23. Lasa L, Ayuso-Mateos JL, Vazquez-Barquero JL, DiezManrique FJ, Dowrick CF. The use of the Beck Depression Inventory to screen for depression in the general population: a preliminary analysis. J Affect Disord. 2000;57(1-3):261-265.

24. Hisli N. A study on the validity of Beck Depression Inventory. J Psychol. 1988;6:118-122.

25. Buysse DJ, Reynolds CF, 3rd, Monk TH, Berman SR, Kupfer DJ. The Pittsburgh Sleep Quality Index: a new instrument for psychiatric practice and research. Psychiatry Res. 1989;28(2):193-213.

26. Agargun MY, Kara H, Anlar O. Pittsburgh uyku kalitesi indeksinin gecerligi ve guvenirligi. Turk Psikiyatri Dergisi. 1996; 7:107-115.

27. Taylor-Gjevre RM, Gjevre JA, Nair B, Skomro R, Lim HJ. Components of Sleep Quality and Sleep Fragmentation in Rheumatoid Arthritis and Osteoarthritis. Musculoskeletal Care. 2011.

28. Cakirbay H, Bilici M, Kavakci O, Cebi A, Guler M, Tan U. Sleep quality and immune functions in rheumatoid arthritis patients with and without major depression. Int J Neurosci. 2004;114(2):245-256.

29. Belza BL. Comparison of self-reported fatigue in rheumatoid arthritis and controls. J Rheumatol. 1995;22(4):639-643.

30. Drewes AM, Jennum P, Andreasen A, Sjul A, Nielsen KD. Self-reported sleep disturbances and daytime complaints in women with fibromyalgia and rheumatoid arthritis. J Musculoskel Pain. 1994;2(1):15-31.

31. Goldenberg DL. Fatigue in rheumatic disease. Bull Rheum Dis. 1995;44(1):4-8.

32. Wells G, Li T, Maxwell L, Maclean R, Tugwell P. Responsiveness of patient reported outcomes including fatigue, sleep quality, activity limitation, and quality of life following treatment with abatacept for rheumatoid arthritis. Ann Rheum Dis. 2008;67(2):260-265.

33. Yount S, Sorensen MV, Cella D, Sengupta N, Grober J, Chartash EK. Adalimumab plus methotrexate or standard therapy is more effective than methotrexate or standard therapies alone in the treatment of fatigue in patients with active, inadequately treated rheumatoid arthritis. Clin Exp Rheumatol. 2007;25(6):838-846.

34. Crosby LJ. Factors which contribute to fatigue associated with rheumatoid arthritis. J Adv Nurs. 1991;16(8):974981.

35. Houssien DA, McKenna SP, Scott DL. The Nottingham Health Profile as a measure of disease activity and outcome in rheumatoid arthritis. Br J Rheumatol. 1997;36(1):69-73.

36. Mahowald MW, Mahowald ML, Bundlie SR, Ytterberg SR. Sleep fragmentation in rheumatoid arthritis. Arthri- tis Rheum. 1989;32(8):974-983.

37. Hewlett S, Cockshott Z, Byron M, Kitchen K, Tipler S, Pope D, Hehir M. Patients' perceptions of fatigue in rheumatoid arthritis: overwhelming, uncontrollable, ignored. Arthritis Rheum. 2005;53(5):697-702.

38. Rupp I, Boshuizen HC, Dinant HJ, Jacobi CE, van den Bos GA. Disability and health-related quality of life among patients with rheumatoid arthritis: association with radiographic joint damage, disease activity, pain, and depressive symptoms. Scand J Rheumatol. 2006;35(3):175-181.

39. Hakkinen A, Kautiainen H, Hannonen P, Ylinen J, Arkela-Kautiainen M, Sokka T. Pain and joint mobility explain individual subdimensions of the health assessment questionnaire (HAQ) disability index in patients with rheumatoid arthritis. Ann Rheum Dis. 2005;64(1):59-63.

40. Prajs K, Flicinski J, Brzosko I, Przepiera-Bedzak H, Ostanek L, Brzosko M. [Quality of life and activity of disease in patients with rheumatoid arthritis]. Ann Acad Med Stetin. 2006;52(Suppl 2):39-43.

41. Prajs K. [The quality of life of patients who suffer from rheumatoid arthritis in reference to their physical fitness and mental state]. Ann Acad Med Stetin. 2007;53(2):7282.

42. Katz PP, Yelin EH. Activity loss and the onset of depressive symptoms: do some activities matter more than others? Arthritis Rheum. 2001;44(5):1194-1202.

43. Kronenberg F, Cote LJ, Linkie DM, Dyrenfurth I, Downey JA. Menopausal hot flashes: thermoregulatory, cardiovascular, and circulating catecholamine and LH changes. Maturitas. 1984;6(1):31-43.

44. Cignarelli M, Cicinelli E, Corso M, Cospite MR, Garruti G, Tafaro E, Giorgino R, et al. Biophysical and endocrine-metabolic changes during menopausal hot flashes: increase in plasma free fatty acid and norepinephrine levels. Gynecol Obstet Invest. 1989;27(1):34-37.

45. Bourguignon $C$, Labyak SE, Taibi D. Investigating sleep disturbances in adults with rheumatoid arthritis. Holist Nurs Pract. 2003;17(5):241-249.

46. Baumgartner H, Hohmeister R, Blumenberg-Novoselac $\mathrm{N}$. An observer-blind crossover study to compare the efficacies of flurbiprofen, indomethacin and naproxen given orally and rectally in the relief of night pain and morning stiffness due to rheumatoid arthritis. J Int Med Res. 1988;16(3):189-196.

47. Vasanthakumar V, Haslock I. The effects of differing pharmaceutical preparations of indomethacin on night pain and morning stiffness in patients with rheumatoid arthritis. Curr Med Res Opin. 1987;10(9):592-595.

48. Lavie P, Nahir M, Lorber M, Scharf Y. Nonsteroidal antiinflammatory drug therapy in rheumatoid arthritis patients. Lack of association between clinical improvement and effects on sleep. Arthritis Rheum. 1991;34(6):655659. 
49. Wells G, Li T, Tugwell P. Investigation into the impact of abatacept on sleep quality in patients with rheumatoid arthritis, and the validity of the MOS-Sleep questionnaire Sleep Disturbance Scale. Ann Rheum Dis. 2010;69(10):1768-1773.

50. Ruperto N, Lovell DJ, Li T, Sztajnbok F, GoldensteinSchainberg C, Scheinberg M, Penades IC, et al. Abatacept improves health-related quality of life, pain, sleep quality, and daily participation in subjects with juvenile idiopathic arthritis. Arthritis Care Res (Hoboken). 2010;62(11):1542-1551.

51. Bixler EO, Kales A, Jacoby JA, Soldatos CR, Vela-Bueno A. Nocturnal sleep and wakefulness: effects of age and sex in normal sleepers. Int J Neurosci. 1984;23(1):3342. 